\title{
Fluorimetric determination of hydrogen peroxide production by the haemocytes of the wax moth Galleria mellonella (Lepidoptera: Pyralidae)
}

\author{
ONDŘEJ VAŠÍČEK ${ }^{1}$, IVANA PAPEŽÍKOVÁ ${ }^{1}$ and PAVEL HYRŠL ${ }^{2}$
}

\author{
'Department of Free Radical Pathophysiology, Institute of Biophysics, Academy of Sciences of the Czech Republic, \\ Královopolská 135, 61265 Brno, Czech Republic; e-mails: ondrej.vasicek@ibp.cz, papezikovai@email.cz \\ ${ }^{2}$ Department of Animal Physiology and Immunology, Institute of Experimental Biology, Faculty of Science, Masaryk University, \\ Kotlářská 2, 611 37, Brno, Czech Republic; e-mail: hyrsl@mail.muni.cz
}

Key words. Lepidoptera, Pyralidae, Galleria mellonella, chemiluminescence, fluorescence, hydrogen peroxide, reactive oxygen species, Amplex Red, haemocytes, phagocytes, leukocytes, phagocytosis

\begin{abstract}
The aim of this study was to investigate whether haemocytes of Galleria mellonella (Lepidoptera: Pyralidae) larvae produce reactive oxygen species (ROS) like human blood phagocytes. The production of ROS was measured first using luminolenhanced chemiluminescence of un-stimulated and stimulated (four activators with different modes of action) haemolymph or isolated haemocytes. However, spontaneous and activated production of ROS remained at the background level. In subsequent experiments an ultrasensitive fluorescence method using Amplex Red reagent to detect hydrogen peroxide $\left(\mathrm{H}_{2} \mathrm{O}_{2}\right)$ was used. After optimization, Amplex Red was successfully used for determining $\mathrm{H}_{2} \mathrm{O}_{2}$ production by both un-stimulated and stimulated haemocytes. To determine the affect of $\mathrm{pH}$ and ions on the measurement, several diluent solutions were tested. This revealed that $\mathrm{Ca}^{2+}$ and $\mathrm{Mg}^{2+}$ ions are less important for the reaction in insect than mammalian cells. Among the activators tested, phorbol myristate acetate (PMA) and calcium ionophore (Ca-I) had the best stimulatory effect on insect samples, while opsonised zymosan particles (OZP) was the best activator for human phagocytes. In conclusion, the haemocytes of $G$. mellonella produce $\mathrm{H}_{2} \mathrm{O}_{2}$ as an important innate immunity factor, but under different conditions and in different amounts, which probably results in them being less effective in killing microbes than human phagocytes.
\end{abstract}

\section{INTRODUCTION}

The immune responses of insects to invading pathogens can be divided into cellular (also called hemocytic) and humoral. Both protect insects against invaders that have overcome the physical barriers posed by the insects' integument, mid-gut epithelium and peritrophic membrane (Elrod-Erickson et al., 2000). Cellular responses, characterized by a direct interaction of haemocytes with antigens, involve phagocytosis, microaggregation, nodulation and encapsulation. Humoral defence involves the action of molecules constitutively present in tissues and haemolymph, such as lectins or lysozymes, induced synthesis of antimicrobial peptides (AMPs) and activation of the phenoloxidase (PO) cascade, while coagulation cascades involve both cellular and humoral mechanisms (Theopold et al., 2002). The insect immune response has a number of structural and functional similarities to the innate immune response of mammals (e.g. Vilmos \& Kurucz, 1998; Salzet 2001; Kavanagh \& Reeves, 2004, 2007; Wang et al., 2010). The mechanism and probably also the proteins involved in the production of superoxide in insect haemocytes are similar to those involved in the NADPH oxidase-induced superoxide production in human neutrophils (Bergin et al., 2005). Insect haemolymph contains haemocytes, which function in a manner similar to that of the phagocytes of humans (Ratcliffe, 1993). At least six types of haemocytes are present in insects (Boman \& Hultmark, 1987), and plasmatocytes and granulocytes are the most abundant types of phagocytic cells. Production of ROS, as an important tool for oxidative killing of invading pathogens during phagocytosis, has been detected in some insects, other invertebrates and mammals; there is also evidence that both $\mathrm{O}_{2}^{-}$(superoxide) (Glupov et al., 2001) and its dismutation product, $\mathrm{H}_{2} \mathrm{O}_{2}$, are present in plasmatocytes of Drosophila melanogaster larvae (Nappi \& Vass,
1998) and G. mellonella (Slepneva et al., 1999; Krishnan et al., 2008). The similarities between the oxidative burst pathways of insect haemocytes and mammalian neutrophils raise the possibility that the complexes that generate ROS might also contain homologous components. Recent reports provide evidence of the involvement of proteins homologous to human neutrophil p47phox and p67phox (Banfi et al., 2003; Geiszt et al., 2003). In this study Amplex Red reagent, an ultrasensitive method for detecting the production of $\mathrm{H}_{2} \mathrm{O}_{2}$, is used to provide evidence of $\mathrm{H}_{2} \mathrm{O}_{2}$ production by the haemocytes of $G$. mellonella larvae.

\section{MATERIAL AND METHODS}

\section{Buffers and reagents}

Three buffers were used in this study: Hank's balanced salt solution (HBSS, in mmol/1: $\mathrm{NaCl} \mathrm{140,} \mathrm{KCl} 5.40, \mathrm{NaHCO}_{3} 4.17$, $\mathrm{CaCl}_{2} 1.26, \mathrm{MgSO}_{4} \cdot 7 \mathrm{H}_{2} \mathrm{O} 0.81, \mathrm{KH}_{2} \mathrm{PO}_{4} 0.44, \mathrm{Na}_{2} \mathrm{HPO}_{4} \cdot 12 \mathrm{H}_{2} \mathrm{O}$ 0.36 , glucose $5.05 ; \mathrm{pH} 7.4$ ), physiological solution for humans (PS) $(0.9 \%$ solution of $\mathrm{NaCl}, \mathrm{pH} 5.5)$ and insect saline (IS, in $\mathrm{mmol} / \mathrm{l}: \mathrm{KCl} 30, \mathrm{NaHCO}_{3} 5, \mathrm{CaCl} 2 \mathrm{H}_{2} \mathrm{O} 8, \mathrm{MgCl} 6 \mathrm{H}_{2} \mathrm{O} 15$, $\mathrm{KH}_{2} \mathrm{PO}_{4} 5$ and sucrose 220, $\mathrm{pH}$ 6.6). Phenylthiourea (PTU) and Amplex Red reagent (10-acetyl-3,7-dihydroxyphenoxazine) were purchased from Sigma (USA), luminol (dissolved in borate buffer, $\mathrm{pH}=9.0$ ) from Molecular Probes (USA) and L-012 (stock solution prepared in borate buffer) from Wako Pure Chemical Industry (Japan). Dextran-T500 (Pharmacia, Sweden) and telebrix N 300 (Léčiva, Czech Republic) were used for isolating leucocytes. Zymosan particles (ZP - Zymosan A from Saccharomyces cerevisiae; Sigma, USA) and zymosan particles opsonised by human serum (OZP), phorbol-12-myristate-13acetate (PMA; Sigma, USA), N-formyl-methionyl-leucylphenylalanin (fMLP) or calcium ionophore (Ca-I A23187; Sigma, USA) were used as activators of ROS production. All 


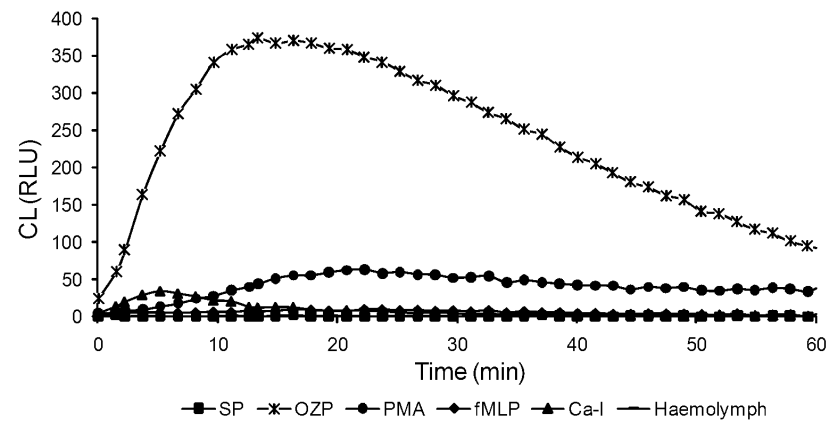

Fig. 1. The kinetics of the chemiluminescence (CL) reaction recorded during ROS production by human blood and haemolymph. Production by blood that occurred spontaneously (SP) or after stimulation with opsonised zymosan particles (OZP), phorbol-12-myristate-13-acetate (PMA), N-formyl-methionylleucyl-phenylalanin (fMLP) or calcium ionophore (Ca-I) was measured. Production by haemolymph was measured under the same conditions, but only one curve corresponding to all background level results is displayed.

other chemicals were purchased from local distributors, and all the chemicals and reagents used were of reagent grade and the highest purity.

\section{Isolation of haemocytes from insects}

Galleria mellonella L. (Lepidoptera: Pyralidae) larvae (VII ${ }^{\text {th }}$ instar, 3-4 days old, $165.32 \pm 2.4 \mathrm{mg}$ ) were obtained from laboratory cultures maintained on an artificial diet (Haydak, 1936) and kept at $28 \pm 1{ }^{\circ} \mathrm{C}$ in constant darkness. Haemolymph with haemocytes from larvae was collected by amputation of a proleg and pooled in a cooled tube containing several crystals of PTU. The haemocytes in haemolymph were diluted with buffers (HBSS, IS or PS) and the haemocytes were separated from haemolymph by centrifugation $\left(500 \times \mathrm{g}, 5 \mathrm{~min}\right.$ at $\left.4^{\circ} \mathrm{C}\right)$ prior to assay. Haemocyte counts were determined using a Bürker's chamber and adjusted (using HBSS, IS or PS) to a final concentration of $1.5 \times 10^{6}$ cells/sample (15-fold higher concentration than in human blood phagocytes).

\section{Isolation of leucocytes from human blood}

The heparinized blood (Na-heparin, $50 \mathrm{IU} / \mathrm{ml}$ ) from healthy volunteers was obtained by antecubital venepuncture. Erythrocytes were removed after $1 \mathrm{~h}$ sedimentation in dextran/telebrix separation solution. For isolation of all the leucocytes, the leucocyte rich plasma (buffy coat) was centrifuged $\left(300 \times \mathrm{g}, 20^{\circ} \mathrm{C}\right.$, $5 \mathrm{~min}$ ). The pellet was exposed to the hypotonic lysis of contaminating erythrocytes; the rest of the leucocytes were washed in HBSS and the total leucocyte counts adjusted to $1 \times 10^{6}$ cells $/ \mathrm{ml}$.

\section{Chemiluminescence assay}

The chemiluminescence (CL) of haemocytes and human whole blood were measured in duplicate using the microtitre plate luminometer LM-01T (Immunotech, Czech Republic) according to Pavelkova \& Kubala (2004). Each reaction mixture contained $1 \times 10^{5}$ leucocytes or $1.5 \times 10^{6}$ haemocytes diluted in HBSS, IS or PS (appropriate solution), $1 \mathrm{mM}$ luminol or $40 \mu \mathrm{M}$ $\mathrm{L}-012$ and $25 \mu \mathrm{l}$ of one of the activators (OZP in a concentration of $62.5 \mu \mathrm{g} / \mathrm{ml}$ or ZP of $62.5 \mu \mathrm{g} / \mathrm{ml}$ for haemocytes, PMA in a concentration of $0.81 \mu \mathrm{g} / \mathrm{ml}$, CaI in a concentration of 9.55 $\mu \mathrm{g} / \mathrm{ml}$ or fMLP in a concentration of $1.14 \mu \mathrm{g} / \mathrm{ml}$ ). Preparation of the stock solutions of luminol and oxidative burst activators follows Pavelkova \& Kubala (2004). HBSS, PS or IS were used to adjust the total reaction volume to $250 \mu 1$.

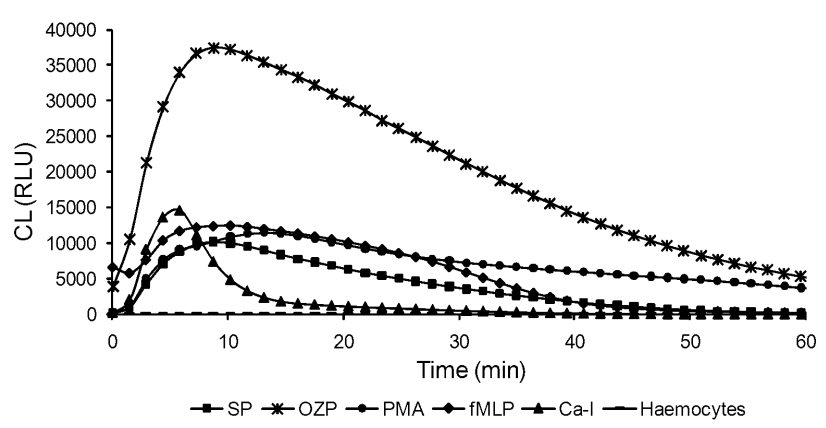

Fig. 2. The kinetics of the chemiluminescence $(\mathrm{CL})$ reaction recorded during ROS production by isolated leucocytes and haemocytes. Production by leucocytes, resuspended in HBSS buffer with luminol that occurred spontaneously (SP) or after stimulation with opsonised zymosan particles (OZP), phorbol12-myristate-13-acetate (PMA), N-formyl-methionyl-leucylphenylalanin (fMLP) or calcium ionophore (Ca-I) was measured. Production by haemocytes was measured under the same conditions, but only one curve corresponding to all background level results is displayed.

\section{Fluorescence assay}

In the presence of peroxidase, the Amplex Red reagent reacts with $\mathrm{H}_{2} \mathrm{O}_{2}$ in a $1: 1$ stoichiometry to produce the red-fluorescent oxidation product, resorufin. Amplex Red solution ( $5 \mu 1$ of 10 $\mu \mathrm{M}$, stock solution in DMSO) was added to $10 \mathrm{ml}$ of the appropriate solution with 10 units of horseradish peroxidase (HRP). The fluorescence (FL) of haemocytes or human leucocytes was measured using the microtitre plate fluorimeter Infinite 200 (Austria) at $585 \mathrm{~nm}$. Firstly the number of haemocytes was optimized in a ratio $0.5-2 \times 10^{6}$ haemocytes per sample and for all subsequent experiments the concentration $1.5 \times 10^{6}$ haemocytes per sample was selected. Human leucocytes at a concentration of $1 \times 10^{5}$ per sample were used as a positive control. Each reaction mixture consisted of a total volume of $500 \mu$ l containing leucocytes or haemocytes (diluted in appropriate solution with Amplex Red) and $25 \mu 1$ of one of the activators and was incubated for $20 \mathrm{~min}$ in the dark.

\section{Statistical analysis}

The results from the fluorescence assay are expressed as the mean \pm standard error (SEM), $n=4$. The level of significance was analysed by one-way analysis of variance (ANOVA), followed by a Tukey's test, values after activation within one buffer group were compared to spontaneous fluorescence.

\section{RESULTS}

\section{Determination of ROS by chemiluminescence}

In the first set of experiments the production of ROS by haemolymph or plasma (e.g. with or without haemocytes) was measured. Whole human blood was used as a positive control. No CL signal was detected in the haemolymph although several different diluents (Hank's balanced salt solution - HBSS, physiological solution - PS or insect saline - IS) and activators (zymosan particles - ZP, phorbol-12-myristate-13-acetate PMA, N-formyl-methionyl-leucyl-phenylalanin - fMLP or calcium ionophore - Ca-I) in various concentrations were used. In contrast, human blood shows a typical kinetic reaction (Fig. 1) with opsonised zymosan particles (OZP) as the best activator.

Subsequently haemocytes were isolated to eliminate potential interference with other haemolymph components. Similarly, leucocytes from whole human blood were isolated as a positive control (Fig. 2). Also under these conditions, the CL signal for 


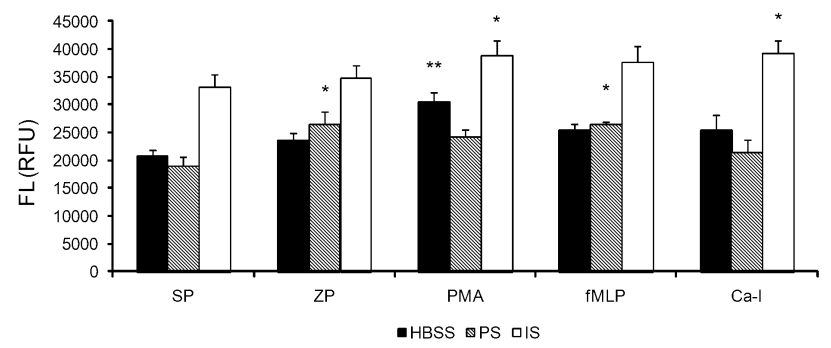

Fig. 3. Fluorescence (FL) determination of $\mathrm{H}_{2} \mathrm{O}_{2}$ production by haemocytes using different diluents (Hank's balanced salt solution - HBSS, physiological solution - PS, insect saline - IS) and activators: zymosan particles (ZP), phorbol-12-myristate13-acetate (PMA), N-formyl-methionyl-leucyl-phenylalanin (fMLP) or calcium ionophore (Ca-I). Data are expressed as mean \pm SEM, $\mathrm{n}=5$, significance level when compared with spontaneous FL (SP) $* \mathrm{p}=0.05, * * \mathrm{p}=0.01$

haemocytes remains at the background level in contrast to the ROS production by leucocytes, which show typical kinetics for individual activators (Fig. 2), and again OZP resulted in the greatest activation. To increase the sensitivity of the method, a stronger luminophor L-012 was also tested, however no CL signal was detected from haemocytes.

\section{Determination of hydrogen peroxide by flourescence}

Due to the negative results for ROS determination in haemocytes by CL, fluorescence was used as a more sensitive method in subsequent experiments.

The $\mathrm{H}_{2} \mathrm{O}_{2}$ production by $G$. mellonella haemocytes after incubation with Amplex red in different buffers and after activation by different activators is shown in Fig. 3 . The best $\mathrm{H}_{2} \mathrm{O}_{2}$ production by spontaneous or activated haemocytes was detectable using IS buffer ( $\mathrm{pH}$ 6.6), otherwise the FL signal was reduced significantly by an average of $32 \%$ with HBSS (pH 7.4, $\mathrm{p}<$ 0.01 , only PMA $\mathrm{p}<0.05$ ) or $39 \%$ with PS (pH 7.4, without $\mathrm{Ca}^{2+}$ and $\mathrm{Mg}^{2+}$ ions, $\mathrm{p}<0.01$ ). Two activators show similar significant $(\mathrm{p}<0.05)$ activation of haemocytes in IS (PMA $38908 \pm$ 2570 RFU and Ca-I $39394 \pm 2087$ RFU) compared with spontaneous $\mathrm{H}_{2} \mathrm{O}_{2}$ production (33287 $\pm 2192 \mathrm{RFU}$, Fig. 4), while that of $\mathrm{ZP}(34819 \pm 2278 \mathrm{RFU})$ and fMLP $(37628 \pm 2859 \mathrm{RFU}) \mathrm{did}$ not differ from spontaneous $\mathrm{H}_{2} \mathrm{O}_{2}$ level. Because the effect of activators on haemocytes was very small compared to human leucocytes, one half or $2-4 \times$ higher concentrations were also used to verify the optimal concentration, but $\mathrm{H}_{2} \mathrm{O}_{2}$ production decreased (low concentration of activator or higher toxicity at higher concentrations, data not shown).

The same measurement was repeated with human leucocytes (Fig. 4). In this case the highest $\mathrm{H}_{2} \mathrm{O}_{2}$ production was detected in spontaneous or activated leucocytes diluted in HBSS, the FL signal was significantly $(\mathrm{p}<0.01)$ reduced by an average of $70 \%$ when PS without $\mathrm{Ca}^{2+}$ and $\mathrm{Mg}^{2+}$ ions and a different $\mathrm{pH}$ was used, similarly $(\mathrm{p}<0.01)$ IS buffer (containing $\mathrm{Ca}^{2+}$ and $\mathrm{Mg}^{2+}$ ions, but lower $\mathrm{pH}$ than HBSS) caused a reduction in the FL signal by an average of $60 \%$. All activators show significant $(p<0.01)$ activation of leucocytes in HBSS compared with spontaneous $\mathrm{H}_{2} \mathrm{O}_{2}$ production $(3635 \pm 417 \mathrm{RFU})$. Among the activators, the best stimulatory effect on human leucocytes was obtained using OZP $(31482 \pm 1179$ RFU) followed by PMA (11904 \pm 748 RFU), fMLP (10577 \pm 1153 RFU) and Ca-I (7887 $\pm 552 \mathrm{RFU})$.

In summary, fluorescence and Amplex Red reagent was more sensitive for measuring insect haemocyte $\mathrm{H}_{2} \mathrm{O}_{2}$ production than luminometry. Elicitors resulted in different levels of haemocyte

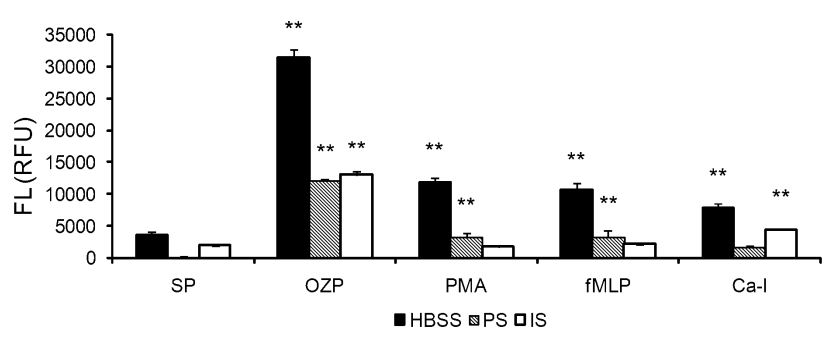

Fig. 4. Fluorescence (FL) determination of $\mathrm{H}_{2} \mathrm{O}_{2}$ production by human leucocytes using different diluents (Hank's balanced salt solution - HBSS, physiological solution - PS, insect saline - IS) and activators: opsonised zymosan particles (OZP), phorbol-12-myristate-13-acetate (PMA), N-formyl-methionylleucyl-phenylalanin (fMLP) or calcium ionophore (Ca-I). Data are expressed as mean \pm SEM, $n=4$, significance level when compared with spontaneous FL (SP) $* * \mathrm{p}=0.01$.

activation, but future optimization is needed to confirm these differences.

\section{DISCUSSION}

Previous studies have reported the production of the superoxide radical (Bergin et al., 2005; Slepneva et al., 1999) and nitric oxide (Krishnan et al., 2006) by G. mellonella. This study focused on the detection of $\operatorname{ROS}\left(\mathrm{H}_{2} \mathrm{O}_{2}\right.$ mainly) in $G$. mellonella haemocytes using common chemiluminescence and fluorescence.

Firstly chemiluminescence using luminol as luminophor was used for ROS detection in haemocytes with human blood as a positive control. Activators with different modes of action were applied to enhance the CL signal (Hoidal et al., 1978; Lad et al., 1985; Pohl et al., 1990). ZP or OZP particles are recognized by phagocyte receptors resulting in the activation of signal pathways including proteinkinase C (PKC), which catalyse endogenous protein phosphorylation followed by activation of NADPH-oxidase (the key enzyme of the respiratory burst). fMLP activator is a bacterial chemotactic polypeptide that activates signal pathways in connection with PKC and NADPHoxidase by specific receptor binding. PMA passes through the cytoplasmatic membrane and directly activates PKC. Calcimycin A23187 (CaI) both increases intracellular calcium and activates PKC. However, no CL signal from haemolymph was detected when these two activators were used. In contrast, stimulated human blood phagocytes produced detectable levels of ROS. Haemocytes and blood phagocytes, respectively, were then isolated because components of haemolymph can interfere with the reaction (Dubovskii et al., 2010). A concentration of $1.5 \times 10^{6}$ per sample (15-fold higher amount of haemocytes than human leucocytes) was selected for subsequent measurements and human leuocytes were again used as a positive control. However, under these conditions the signal from isolated haemocytes remained at the background level in contrast to the ROS production by leucocytes. Additional approaches included using greater numbers of haemocytes, different buffers, more sensitive luminophor (L-012 instead of luminol - Daiber et al., 2004) and a higher concentration of activator. None of these changes led to detectable ROS production by haemocytes, which confirm previous luminometry results on Bombyx mori (Hyršl et al., 2004).

Fluorimetric ROS detection was used because it is a more sensitive method as it measures accumulated ROS rather than the level at one point in time, like CL. This method was used successfully e.g. for the measurement of the oxidative burst in lobster haemocytes (Moss \& Allan, 2006). The fluorescent rea- 
gent Amplex red was selected since it is a commonly used reagent for hydrogen peroxide $\left(\mathrm{H}_{2} \mathrm{O}_{2}\right)$ production in mammalian cells, but there is only one report of it having been used for insects (Krishnan et al., 2008). Isolated haemocytes and human leucocytes showed a detectable fluorescence signal. After optimizing the number of haemocytes to a range of $0.5-2 \times 10^{6}$, a final concentration of $1.5 \times 10^{6}$ per sample was selected, because measured values of $\mathrm{H}_{2} \mathrm{O}_{2}$ production were comparable with that produced by leucocytes (but the number of leucocytes was 15 -fold lower). Using these conditions different buffers and activators were tested. HBSS is the standard buffer designed for human blood cells with pH 7.4 and contains $\mathrm{Ca}^{2+}$ and $\mathrm{Mg}^{2+}$ ions, which are necessary for NADPH oxidase activation with subsequent ROS production (Brookes et al., 2004; Konrad et al., 2004; Touyz, 2004). Other buffers used include PS without $\mathrm{Ca}^{2+}$ and $\mathrm{Mg}^{2+}$ ions, with $\mathrm{pH} 5.5$ and IS buffer designed for insect haemocytes, with $\mathrm{pH}$ 6.6, which is similar to the $\mathrm{pH}$ of haemolymph, 6.4-6.8 (Florkin \& Jeuniaux, 1974; Mullins, 1985). Composition of IS is similar to HBSS as it also contains $\mathrm{Ca}^{2+}$ and $\mathrm{Mg}^{2+}$ ions.

Insect haemocytes produced the highest amount of $\mathrm{H}_{2} \mathrm{O}_{2}$ in IS buffer. In HBSS or PS the production of ROS was greatly decreased. These results indicate that haemocytes are not equally sensitive to $\mathrm{pH}$ changes and the presence of $\mathrm{Ca}^{2+}$ and $\mathrm{Mg}^{2+}$ ions is not as important as in human leucocytes. The stimulatory effects of different activators are not as pronounced as in human leucocytes, but statistical analysis confirmed significant differences in the activation of $\mathrm{H}_{2} \mathrm{O}_{2}$ production when using PMA and Ca-I (in the case of PMA also using HBSS buffer). Thus activator concentrations optimized for human cells (Pavelkova \& Kubala 2004) are not suitable for insect haemocytes, thus different concentrations were tested. However, both lower and higher concentrations (one half and double, respectively) resulted in a lower $\mathrm{H}_{2} \mathrm{O}_{2}$ production. Higher concentrations of activators can be also be toxic for cells (Przygodzki et al., 2005) thus the concentration used in this study seems to be optimal for haemocytes.

For human leucocytes the highest production of $\mathrm{H}_{2} \mathrm{O}_{2}$ was observed using HBSS, which was expected as it is a special preparation for mammalian cells. Using IS and PS buffers with different compositions and $\mathrm{pHs}$ the $\mathrm{H}_{2} \mathrm{O}_{2}$ production led to a decrease in the signal. Thus, for human leucocytes it is more important that the $\mathrm{pH}$ is 7.4 and $\mathrm{Ca}^{2+}$ and $\mathrm{Mg}^{2+}$ ions are present. Regarding activators, the one with the best stimulatory effect on $\mathrm{H}_{2} \mathrm{O}_{2}$ production was OZP followed by PMA, fMLP and Ca-I.

To conclude, this study presents an ultrasensitive method, which uses Amplex Red reagent for determining $\mathrm{H}_{2} \mathrm{O}_{2}$ in insect haemocytes. The results show that the haemocytes of G. mellonella produced a low level of ROS. It is likely that ROSproduction contributes less to direct bactericidal activity than to signalling pathways regulating immune reactions.

ACKNOWLEDGEMENT. This research was funded by a research grant from the Grant Agency of Czech Republic (GA206/09/P470). We are grateful to U. Theopold (Stockholm University, Sweden) for his useful comments on this article.

\section{REFERENCES}

Banfi B., Clark R.A., Steger K. \& Krause K.H. 2003: Two novel proteins activate superoxide generation by the NADPH oxidase NOX1. J. Biol. Chem. 278: 3510-3513.

Bergin D., Reeves E.P., Renwick J., Wientues F.B. \& Kavanagh K. 2005: Superoxide production in Galleria mellonella hemocytes: identification of proteins homologous to the NADPH oxidase complex of human neutrophils. Infect. Immun. 73: $4161-4170$.
Boman H.G. \& Hultmark D. 1987: Cell-free immunity in insects. Annu. Rev. Microbiol. 41: 103-126.

Brookes P.S., Yoon Y., Robotham J.L., Anders M.W. \& Sheu S.S. 2004: Calcium, ATP, and ROS: a mitochondrial lovehate triangle. Am. J. Physiol. Cell Physiol. 287: C817-C833.

Daiber A., August M., Baldus S., Wendt M., Oelze M., Sydow K., Kleschyov A.L. \& Munzel T. 2004: Measurement of $\mathrm{NAD}(\mathrm{P}) \mathrm{H}$ oxidase-derived superoxide with the luminol analogue L-012. Free Radic. Biol. Med. 36: 101-111.

Dubovskit I.M., Grizanova E.V., Chertkova E.A., Slepneva I.A., Komarov D.A., Vorontsova Ya.L. \& Glupov V.V. 2010: Generation of reactive oxygen species and activity of antioxidants in larva hemolymph of galleria mellonella (L.) (Lepidoptera: Pyralidae) at development of process of encapsulation. J. Evol. Biochem. Physiol. 46: 35-43.

Elrod-Erickson M., Mishra S. \& Schneider D. 2000: Interactions between the cellular and humoral immune responses in Drosophila. Curr. Biol. 10: 781-784.

Florkin M. \& Jeuniaux C. 1974: Hemolymph: Composition. In: Rockstein M. (ed.): The Physiology of Insecta. 2nd ed. Vol. V. Academic Press, New York, pp. 255-308.

Geiszt M., Lekstrom K., Witta J. \& Leto T.L. 2003: Proteins homologous to $\mathrm{p} 47 \mathrm{phox}$ and $\mathrm{p} 67$ phox support superoxide production by $\mathrm{NAD}(\mathrm{P}) \mathrm{H}$ oxidase 1 in colon epithelial cells. $J$. Biol. Chem. 278: 20006-20012.

Glupov V.V., Khvoshevskaya T.L., Lovinskaya Y.L., Dubovski I.M., Martemyanov V.V. \& SoKolova J.Y. 2001: Application of the nitroblue tetrazolium reduction method for studies on the production of reactive oxygen species in insect haemocytes. Cytobios 106: 165-178.

HAYDAK M.H. 1936: A food for rearing laboratory insect. $J$. Econ. Entomol. 29: 1026.

Hoidal J.R., Reptne J.E., Beall G.D., Rasp F.L. \& White J.G. 1978: The effect of phorbol myristate acetate on the metabolism and ultrastructure of human alveolar macrophages. Am. J. Pathol. 91: 469-482.

Hyršl P., Č́̇̌z M., Kubala L. \& LoJeK A. 2004: Silkworm (Bombyx mori) hemocytes do not produce reactive oxygen metabolites as a part of defense mechanisms. Folia Microbiol. 49: 315-319.

Kavanagh K. \& ReEves E.P. 2004: Exploiting the potential of insects for the in vivo pathogenicity testing of microbial pathogens. FEMS Microbiol. Rev. 28: 101-112.

KavanaGH K. \& Reeves E.P. 2007: Insect and mammalian innate immune responses are much alike. Microbe 2: 596-599.

Konrad M., Schlingmann K.P. \& Gudermann T. 2004: Insights into the molecular nature of magnesium homeostasis. Am. J. Physiol. Renal. Physiol. 286: F599-F605.

KRISHNAN N., HYRŠL P. \& ŠIMEK V. 2006: Nitric oxide production by hemocytes of larva and pharate prepupa of Galleria mellonella in response to bacterial lipopolysaccharide: Cytoprotective or cytotoxic? Comp. Biochem. Physiol. (C) 142: 103-110.

Krishnan N., Davis A.J. \& Giebultowicz J.M. 2008: Circadian regulation of response to oxidative stress in Drosophila melanogaster. Biochem. Biophys. Res. Commun. 374: 299-303

LAD P.M., Olson C.V. \& SMiley P.A. 1985: Association of the $\mathrm{N}$-formyl-Met-Leu-Phe receptor in human neutrophils with a GTP-binding protein sensitive to pertussis toxin. Proc. Natl. Acad. Sci. USA 82: 869-873.

Moss B. \& Allam B. 2006: Fluorimetric measurement of oxidative burst in lobster hemocytes and inhibiting effect of pathogenic bacteria and hypoxia, J. Shellfish Res. 25: 1051-1058.

Mullins D.E. 1985: Chemistry and physiology of the hemolymph. In Kerkut G.A. \& Gilbert L.I. (eds): Comprehensive 
Insect Physiology, Biochemistry and Pharmacology. Vol. 3. Pergamon Press, Elmsford, NY, pp. 355-400.

NAPPI A.J. \& VASS E. 1998: Melanogenesis and the generation of cytotoxic molecules during insect cellular immune reactions. Pigment Cell Res. 6: 117-126.

Pavelková M. \& Kubala L. 2004: Luminol-, isoluminol- and lucigenin-enhanced chemiluminescence of rat blood phagocytes stimulated with different activators. Luminescence 19: $37-42$.

Pohl P., Antonenko Y.N. \& Yaguzhinsky L.S. 1990: Kinetic properties of cation/ $\mathrm{H}(+)$-exchange: calcimycin (A23187)-mediated $\mathrm{Ca} 2+/ 2 \mathrm{H}(+)$-exchange on the bilayer lipid membrane. Biochim. Biophys. Acta 1027: 295-300.

Przygodzki T., SoKal A. \& BryszewsKa M. 2005: Calcium ionophore A23187 action on cardiac myocytes is accompanied by enhanced production of reactive oxygen species. Biochim. Biophys. Acta 1740: 481-488.

RATCLIFFE N.A. 1993: Cellular defence responses in insects: unresolved problems. In Bechage N.E., Thompson S.N. \& Federace B.A. (eds): Parasites and Pathogens of Insects. Vol. 1. Academic Press, San Diego, CA, pp. 267-304.
SAlzet M. 2001: Vertebrate innate immunity resembles a mosaic of invertebrate immune responses. Trends Immunol. 22: 285-288.

Slepneva I.A., Glupov V.V., Sergeeva S.V. \& Khramtsov V.V. 1999: EPR detection of reactive oxygen species in hemolymph of Galleria mellonella and Dendrolimus superans sibiricus (Lepidoptera) larvae. Biochem. Biophys. Res. Commun. 264: 212-215.

Theopold U., Li D., Fabbri M., Scherfer C. \& Schmidt O. 2002: The coagulation of insect hemolymph. Cell. Mol. Life Sci. 59: 363-372.

Touyz R.M. 2004: Magnesium in clinical medicine. Front Biosci. 9: $1278-1293$.

Vilmos P. \& KuRUCZ E. 1998: Insect immunity: Evolutionary roots of the mammalian innate immune system. Immunol. Lett. 62: 59-66.

Wang Z., Wilhelmsson C., Hyrsl P., Loof Torsten G., Dobes P., Klupp M., Loseva O., Mörgelin M., Ikle J., Cripps R.M., Herwald H. \& Theopold U. 2010: Pathogen entrapment by transglutaminase - a conserved early innate immune mechanism. PLoS Pathogens 6(2): doi:10.1371/journal.ppat. 1000763.

Received October 29, 2010; revised and accepted December 3, 2010 